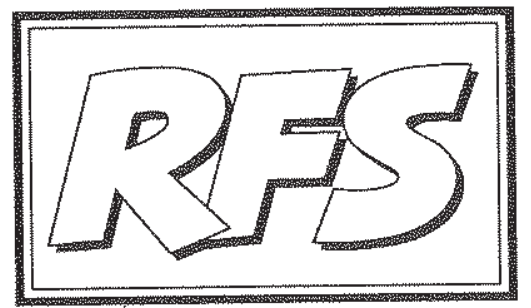

Revista de Fomento Social, 51 (1996), 83-118

\title{
Los principios cooperativos del siglo XXI: Una interpretación crítica
}

El 23 de septiembre de 1995, la Alianza Cooperativa Internacional, máximo órgano de representación de las cooperativas a nivel mundial, aprobó en Manchester (Reino Unido) una declaración sobre la identidad cooperativa que suponía una reformulación de los tradicionales principios cooperativos. En el presente trabajo se realiza una exposición de su contenido y una interpretación de las consecuencias que dichos principios conllevan a partir de las reflexiones realizadas con anterioridad a esta declaración (1) y la experiencia del autor a través de investigaciones realizadas en las cooperativas que hacen realidad estos principios desde su funcionamiento empresarial.

$$
\text { Alfonso Carlos MORALES GUTIÉRREZ }
$$

(*)

(*) Profesor de Economía de la Empresa de la Facultad de Ciencias Económicas y Empresariales - ETEA, de la Universidad de Córdoba.

(1) Algunas de las ideas que se expresan en este trabajo parten de reflexiones breves que 


\section{La nueva formulación de la Alianza Cooperativa Internacional (A.C.I.) como marco de análisis}

Nada hay más práctico que una buena teoría. Hablar de teoría en el cooperativismo es hablar de los tan conocidos principios cooperativos. Los principios cooperativos clásicos tienen su origen en las aportaciones teóricas y prácticas -sistematizadas en sus estatutos- de los 28 socios fundadores de la Rochdale Society of Equitable Pioneers -una cooperativa de consumo- (2). A partir de dichos estatutos, y tras ciertas modificaciones -1845,1854-, se fueron estableciendo de forma tácita los principios básicos de la cooperación, que constituyen al fin y al cabo emanaciones de las doctrinas de diversos pensadores pertenecientes sobre todo, a corrientes filosóficas ligadas al socialismo utópico (FOURIER, OWEN,...).

El congreso internacional de la ACI en Viena (1966) los aprobó en los términos que resumimos a continuación: adhesión voluntaria, un hombre un voto, interés limitado al capital, reparto de los excedentes según la actividad cooperativizada, promoción de la educación entre los socios y, por último, la intercooperación con las restantes cooperativas. El reciente XXXI Congreso de la ACI celebrado en Manchester (1995) ha realizado algunas modificaciones, proponiendo siete principios que denomina de la siguiente forma: adhesión voluntaria y abierta, gestión democrática por parte de los socios, participación económica, autonomía e independencia; educación, formación e información; cooperación entre coope-

fueron publicadas parcialmente en diversas revistas con los siguientes títulos: Morales Gutiérrez, A.C. (1994), “Abrir o cerrar las puertas?, He ahí la cuestión” Economía Social Andaluza, Morales Gutiérrez, A.C. (1994) "Una condición necesaria no suficiente: un socio, un voto" Economía Social Andaluza, n"5, pp. 22-23, Morales Gutiérkez, A.C. (1994)"La caza de mamut y los principios cooperativos sobre el beneficio empresarial", $n^{\circ} 6$, pp. 30-31; Morales Gunérrez, A.C. (1994) "Cooperativistas o cómplices", Andalucía Cooperativa (reproducido con autorización en Vida Cooperativa); Morales Gutrerrez, A.C. (1993) "Nuevo cooperativismo: individuo y capital", Diario Córdoba.

(2) Conviene recordar que la experiencia de Rochdale no es la primera experiencia cooperativa en sentido estricto. Son también muy significativas desde un punto de vista histórico la cooperativa de joyeros de París -cooperativa de trabajo asociado-, las cooperativas de crédito del sistema Raiffesen, ...etc. En todas estas experiencias se realiza una declaración de principios en consonancia con los valores universales de la cooperación pero con peculiaridades específicas según sus diversas formas organizativas. 
rativas; interés por la comunidad. Tanto en 1966 como en 1995 se propone una aplicación gradual de dichos principios según las características y circunstancias de las diferentes realidades cooperativas.

Pretendemos aportar ciertas reflexiones que iluminen la interpretación teórica de los principios, puesto que en algunos casos puede constatarse que su aplicación en el "día a día" de las cooperativas dista tanto de su formulación teórica hasta tal punto que se consideran inaplicables o no responden a la idea que la gente tiene de la cooperación (RAMAEKERS,1984). Para el logro de este propósito hemos organizado nuestra reflexión en cinco partes. En la primera, destacamos la definición y la declaración explícita de valores que se ha producido en el XXXI Congreso de la ACI. En la segunda, realizamos un recorrido a lo largo de los principios cooperativos que, teóricamente, no han cambiado su formulación sugiriendo algunas interpretaciones críticas al respecto. En la tercera y cuarta parte, nos referiremos a aquellos principios que o son nuevos, o sufren modificaciones relevantes. Por último realizaremos una referencia expresa a ciertos principios cooperativos "ausentes", o que son "eludidos" en la declaración institucional de la ACl.

\section{Aportaciones destacables: definición de valores}

Entre los aspectos más destacables de la declaración de Manchester encontramos una definición de cooperativa que subraya como rasgos esenciales, su vinculación a la necesidad de sus socios, el carácter colectivo de su propiedad y su funcionamiento democrático: Una cooperativa es una asociación voluntaria autónoma de personas que se han unido de forma voluntaria para satisfacer sus necesidades y aspiraciones económicas, sociales y culturales en común mediante una empresa de propiedad conjunta y de gestión democrática (3).

También hace la declaración una referencia expresa a los valores. Es la primera vez que se distingue en la $\mathrm{ACl}$ entre valores -cooperativos y éticos-, y principios. Los valores de la cooperación se presentan así: Las cooperativas están basadas en los valores de la autoayuda, la autorresponsabilidad, la democracia, la igualdad, la equidad y la solidaridad. En la tradición de sus

(3) Hemos utilizado como fuente de la declaración de Manchester: INFES, Revista de Economía Social, Octubre, 1995, p.14. 
fundadores, los socios cooperativos hacen suyos los valores éticos de la honestidad, la transparencia, la responsabilidad y la vocación social.

En otro lugar(MORALES, 1992) destacábamos indirectamente dos de los seis valores explicitados ahora por la ACI: la soberanía de la persona -autoayuda, autorrespondabilidad, democracia-y la solidaridad. En efecto, el denominador común en el diseño de este modelo empresarial, y que le aporta su singularidad, es la subordinación del factor capital, que ya no "contrata" a los restantes factores productivos: el capital ha perdido su hegemonía. Es una empresa centrada en el trabajo, en la actividad emprendida en común, en la persona, que en último término, es la que realiza esta actividad. Planteada de esta forma, es la empresa "bumana", la empresa "personalista" por excelencia -en contraposición a la empresa "capitalista"-.

En cuanto al segundo valor cooperativo, nada se entendería en el cooperativismo sin la óptica de la solidaridad. Pero no perdiéndose en la grandilocuencia en su propia palabra, sino como algo vivo que salvaguarda la prioridad del hombre, implicándolo responsablemente en el proyecto, siendo el motor de la actividad imaginativa al facilitar el clima de confianza para que el grupo pueda ser participativo, emprendedor, adaptable y eficaz. La solidaridad en el cooperativismo presenta diversas perspectivas y manifestaciones: en la incorporación a la sociedad, en la distribución de los resultados y percepción de rentas, en la relación de sus socios, con el resto de empresas cooperativas y con su entorno.

Añadiremos un breve comentario a una última aportación significativa realizada en la declaración de Manchester: la explicitación de valores éticos. Nos parece muy oportuna la referencia expresa a valores tales como la transparencia -de vital importancia en las relaciones socios-organos directivos- y la responsabilidad social -estrechamente vinculada al nuevo principio cooperativo de interés por la comunidad-.

\section{Los principios que no cambian en su contenido}

En este apartado nos vamos a referir a cuatro principios "clásicos" del cooperativismo que en la nueva formulación de la $\mathrm{ACl}$ no presentan novedades sustantivas en su contenido, aunque si algunas en su formulación. En concreto los principios que se tratan son el de puertas abiertas, participación democrática, participación económica e intercooperación.

\section{RFS}




\subsection{El principio de puerta abierta}

\section{Formulación de 1.966:}

"La afiliación a una cooperativa

deberá ser voluntaria, al alcance

de todas las personas que puedan

utilizar sus servicios y que están

de acuerdo para asumir las

responsabilidades inherentes a

la calidad de miembro; en las

cooperativas no deben darse

restricciones que no sean naturales

(es decir, impuestas arbitrariamente),

ni ninguna discriminación social,

política, religiosa o racial".

\section{Formulación de 1.995:}

"Las cooperativas son

organizaciones

voluntarias, abiertas a todas

las personas capaces de utilizar

sus servicios y dispuestas a

aceptar las responsabilidades

de ser socio sin discriminación política, religiosa,

racial o de sexo".

La concepción más o menos estricta de este principio conlleva todo tipo de repercusiones, positivas o negativas, dependiendo del tipo de cooperativa (4). Una interpretación literal llevaría a la conclusión de que: a) la cooperativa está obligada a admitir a toda persona que manifieste su voluntad de ser socio y se comprometa a cumplir los estatutos; b) cualquier socio puede salir de la cooperativa cuando lo desee y sin obligación alguna (5).

(4) Diversos autores han estudiado las repercusiones de todo tipo -económicas y jurídicasde la aplicación de este principio. Entre otros: CABALLER, V. (1982); "Aspectos económicos de las altas y bajas de los socios en las cooperativas agrarias". Revista de Economía Política. $\mathrm{n}^{290}$, pp.201-217; Romero, C.; Domingo, J.;(1983) "Análisis económico-financiero del principio de puertas abiertas y del equilibrio a corto plazo en las empresas cooperativas españolas". Revista Española de Financiación y Contabilidad, pp.344-364; Serrano Soldevilla, A.D. (1982); la cooperativa como sociedad abierta. Madrid, Ministerio de Trabajo y Seguridad Social. Servicio de Publicaciones. Madrid.

(5) El principio de puertas abiertas, puede suponer -en su vertiente "negativa" de libertad en la "salida" de socios" una descapitalización de la cooperativa como consecuencia de la retirada de fondos, tanto de las aportaciones como de los beneficios acumulados que el socio posee cuando se da de baja en la cooperativa. Sólo una eficaz protección legal de tal riesgo puede contrarrestar dicho efecto.

\section{ESTUDIOS}


Esta interpretación tradicional del principio de "libre adhesión" "voluntarista" o de puertas abiertas esbozada por ciertos teóricos de la cooperación, ha sido fundamentada en el posible carácter "altruista" de la cooperativa (DOMINGO, 1984).Si por altruismo entendemos "esmero y complacencia en el bien ajeno, aún a costa del propio, y por motivos puramente humanos", dicha interpretación no se sostiene. La propia A.C.I. afirma que: una cooperativa también necesita libertad para modificar su actitud de ingreso sin limitaciones y tener la posibilidad de rechazar solicitudes de ingreso, o expulsar a un socio si los intereses de los demás socios así lo aconsejan. Según esta salvedad de la ACI cambiaría totalmente el contenido semántico de altruismo.

Otra interpretación del principio de puertas abiertas se refiere a las posibilidades de limitar el alta de los socios por razones personalistas de ningún tipo. Así sería un acto contra natura jurídica, absolutamente nulo, toda discriminación por razón política, sexual, de raza, religión o cualquier otra de contenido ideológico y/o personal (DIVAR, 1985). Tal interpretación no sería más que una prolongación de los principios constitucionales generalmente aceptados en la mayor parte de los países y para todo tipo de sociedades. Desde este planteamiento argumentar la vigencia de ese principio como tal, nos parece desacertada.

En resumen, el carácter ideológico del principio de puertas abiertas basado en la no discriminación de la persona como cuestión previa y única para su admisión, se encuentra prácticamente desfasado, pues su aplicación a las diversas realidades cooperativas resulta, o evidente -como es el caso de las cooperativas de consumo-, o inviable -como en las cooperativas de trabajo asociado-. En el fondo la "puerta" está abierta o cerrada independientemente de la existencia del principio y sin mermar para ello su carácter cooperativo.

Sin embargo, podemos realizar una "reformulación del principio" definiéndolo como solidaridad continua es decir, la cooperativa, en su compromiso de generar riqueza, debería admitir nuevos socios, debería tener sus puertas abiertas (6); $y$, en su vertiente negativa, los socios que la abandonen se vinculan a un

(6) Una expresión de esta solidaridad se manifiesta en los datos siguientes del Libro Blanco de la Economía Social (1991) para las cooperativas de trabajo asociado. El incremento medio de trabajadores por cooperativa desde su creación a nivel nacional asciende a casi cinco. Este comportamiento no ha sido homogéneo para todo el territorio nacional con resultados que varian ostensiblemente según las comunidades autónomas. 
compromiso de solidaridad con los que se quedan para no descapitalizar la empresa.

Una práctica anticooperativa, en muchas empresas que jurídicamente figuran como tales, consiste en el establecimiento de aportaciones al capital social que son de hecho inaccesibles para nuevos socios y, por tanto, impiden su incorporación a la sociedad (7). En definitiva se trata de una forma de transgredir el principio de solidaridad para el ingreso y para la salida. Generalmente se manifiesta en cooperativas de trabajo asociado que, una vez alcanzado cierto éxito en la actividad que desarrollan, optan por una política de autofinanciación mediante la incorporación de beneficios al capital social, en lugar de dotar, por ejemplo, reservas que tengan carácter colectivo -irrepartibles- (8). Una vez resuelto el problema de asignación de los recursos financieros, surge la cuestión de cómo dotarse de recursos humanos que mantengan el ritmo de crecimiento o, si es posible, lo incrementen. La estrategia consecuente para sostener la expansión desde la insolidaridad- sería la contratación de mano de obra asalariada.

Al final del proceso habría que plantearse hasta qué punto esta empresa es una cooperativa. El capital, acumulado en manos de unos pocos trabajadores, sigue subordinado al factor trabajo, en concreto a la mano de obra asalariada. La cooperativa pierde así su carácter transformador y se convierte en un instrumento

(7) Según los datos que utilizamos en la elaboración del Libro Blanco de la Economía Social en España (1991) la media de aportación obligatoria al capital social que las cooperativas de trabajo asociado exigen a sus nuevos socios no llega al medio millón de pesetas, con variaciones muy significativas dependiendo de 1 sector al que pertenecen. Superan esta media las cooperativas correspondientes a los siguientes sectores: "Reparaciones" (1.978 miles de ptas.), "Transporte" (1.415), "Maquinaria" (836), "Agrario" (804), "Cuero y Calzado" (795). Con cifras muy por debajo de la media nacional se encuentran los sectores de: "Hostelería" (31) "Comercio al por mayor" (47), "Industria de la Confección" (165), "Industria del Vidrio" (166), "Limpieza" (201).

(8) Esta solidaridad en el terreno financiero quedaba expresada por Bovchez (VARA Miranda, 1985, p. 44-45) de la siguiente foma: el capital social que aumentará así cada año en un quinto de los beneficios, será inealienable; pertenecerá a la a sociación que será declarada indisoluble, y no porque los individuos no puedan marcharse, sino porque la sociedad se convertirá en perpetua mediante la admisión continuada de nuevos miembros... si se hiciera de otra forma, la asociación llegaría a ser semejante a cualquier otra compañía mercantil; sería útil, únicamente para los fundadores, perjudicial para todos aquellos que no hubieran formado parte de ella desde el principio, y acabaría siendo, en las manos de los primeros un medio de explotación. 
de consolidación del sistema liberal-capitalista. Serían pocas, por no decir ningunas, las diferencias con una empresa constituida como una sociedad anónima, o sociedad limitada, con varios socios de participación igualitaria en el capital social.

Ahora bien las cooperativas que adoptan este planteamiento insolidario pueden llegar a una situación crítica: el momento de la jubilación de los socios fundadores. ¿Qué harán entonces? ¿cederán su empresa a trabajadores como ellos -como ocurriría en una cooperativa-? ¿o venderán la empresa en el mercado al mejor postor?. Siguiendo una analogía, la cooperativa que de alguna forma se enfrenta a su fin, como el árbol que va ser talado, se inclinará en la dirección en que creció y se desarrolló...

\subsection{Una condición necesaria, no suficiente: un socio, un voto}

\begin{tabular}{l|l|}
$\begin{array}{l}\text { Formulación de 1.966: } \\
\text { "Las sociedades del }\end{array}$ & Formulación de 1995: \\
cooperativismo son & Las cooperativas son \\
organizaciones democráticas. & organizaciones gestionadas \\
Sus asuntos deberán ser & democráticamente por los \\
administrados por las personas & socios, los cuales participan \\
nombradas, según el & activamente en la fijación de \\
procedimiento adoptado por los & sus políticas y en la toma de \\
miembros, ante los cuales tales & decisiones. Los hombres y mujeres \\
personas son responsables. Los & elegidos para representar y \\
miembros de las asociaciones & gestionar las cooperativas son \\
primarias deberán tener los & responsables ante los socios. En \\
mismos derechos de voto y de & las cooperativas de primer grado, \\
participación en las decisiones & los socios tienen iguales \\
concernientes de su sociedad. & derechos de voto (un socio, \\
En todas las demás sociedades, & un voto), y las cooperativas \\
la administración deberá & de otros grados están también \\
ejercerse sobre una base & organizadas de forma democrática". \\
democrática, bajo una forma & \\
apropiada". &
\end{tabular}


Este principio democrático supone que la organización social de la cooperativa se establece de forma participativa y transparente. Esto implica por lo menos tres condiciones: sólo será posible una administración colegiada; la soberanía social radicará necesariamente en la asamblea de socios y siempre existirá un órgano fiscalizador con facultades inspectoras plenas (DIVAR, 1985).

Se configura la cooperativa como una sociedad "personalista", en contraposición a las fórmulas capitalistas donde es la aportación de capital lo que determina el poder de decisión. En una empresa cooperativa, por el contrario, el poder está en el individuo y no en el capital, según un modelo que potencia el factor humano y relega el capital a simple medio de apoyo.

Este diseño teórico significa, por un lado, una concepción de empresa de futuro, donde la inversión más importante son las personas ya que son la clave para su éxito social y económico. Si en la era industrial, cuando el recurso estratégico lo constituía el capital, el único objetivo eran los beneficios, en la era de la información el recurso estratégico se basa en el conocimiento y en la creatividad. La única forma que tiene la empresa para obtener tan valioso recurso es por medio de su personal, porque es en éste donde se encuentra. En definitiva se trata sencillamente de aceptar que los seres humanos son capaces de crear o de hundir una empresa (NAISBITT, 1986).

Por otro lado, todavía en el plano teórico, la cooperativa representa una plasmación de una concepción del hombre y de la sociedad. "Un socio, un voto" significa en el fondo un forma práctica de traducir en la empresa el principio de igualdad de las personas, más o menos universalmente aceptado, pero "ilógicamente" contradicho cuando se trata de trasplantar ese valor en la empresa. Por eso LAMBERT (1975) afirma que este principio brota del carácter de personas que poseen los miembros de las cooperativas.

Puede afirmarse, sin temor a una generalización excesivamente apresurada, que la mayor parte de los autores coinciden en la aplicación práctica-por lo menos formal- de este principio en las cooperativas de primer grado. Sin embargo, el voto único por persona noes en sí suficiente garantía de una gestión democrática de la sociedad, en especial cuando el número de socios alcanza determinado límite a partir del cual se imposibilita un verdadero contacto personal entre la dirección y los dirigidos. El tamaño es un obstáculo para un funcionamiento democrático en cualquier organización social.

RAMAEKERS (1984) aborda las consecuencias de este problema afirmando 
que por esta falta de control democrático, las cooperativas evolucionarán hacia una concentración del poder en una tecnocracia que acabará por elegir a sus dirigentes entre sus pares. Los síntomas son claros: asambleas de cooperativistas silenciosas -cuando no vacías-, aprobaciones automáticas de las gestiones del consejo rector, y equipos directivos que permanecen inmutables. Además el principio de "un socio, un voto" se sostiene bajo el peligro de que, se necesita una sólida formación y convicción cooperativista para aceptar sin reservas que las desiguales aportaciones capitalistas, que es tanto como decir las desiguales responsabilidades económicas asumidas, no son tenidas en cuenta a la hora de decidir sobre las actividades sociales, asignando el mismo voto al que mucho comprometió y al que apenas aportó.

HESSELLBACH (1975) advierte en este sentido que la participación democrática somete a la cooperativa al riesgo de descapitalización: existe el peligro de que se sacrifique, tarde o temprano, el autofinanciamiento para dar lugar a un consumo más elevado o a más beneficios sociales y este peligro será mayor en las cooperativas de producción que en las empresas privadas. Por tanto, si los cooperativistas no tienen formación suficiente la participación se debilita paulatinamente, el poder se concentra en pocas manos o se incurre en decisiones erróneas.

En bastantes cooperativas agrícolas suele constatarse un hecho patente: de la misma forma que resulta incoherente, desde un planteamiento de soberanía de la persona sobre el capital, asignar la participación social según el capital aportado, resulta también injusto que todos los socios tengan igualdad de derecho a voto cuando participan muy desigualmente en la actividad cooperativizada. Con este argumento los socios con mayor participación en la actividad cooperativizada, reivindican que su poder decisional debe ser mayor; en definitiva, reclaman su derecho a ejercitar un voto plural.

Nuestra posición se basa en el siguiente planteamiento: una cooperativa donde la participación social en la actividad es desigual ha incumplido desde el comienzo un principio que en nuestra opinión, resulta imprescindible para que el cooperativismo cumpla sus fines: la homogeneidad social. Silos socios nocooperativizan su actividad de forma, más o menos, homogénea, su participación en la cooperativa puede verse mediatizada. La reivindicación de poder de los socios será una consecuencia de un origen viciado: una base social desigual.El dinamismo cooperativollevará, portanto, a aumentar más las desigualdades, en lugar de corregirlas.

Pero el problema del ejercicio del principio de gestión democrática no acaba

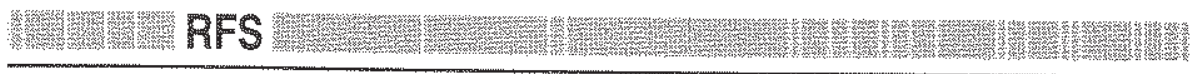


en las cooperativas de primer grado con elevado número de socios; la cuestión se hace mucho más espinosa cuando nos encontramos ante cooperativas de segundo o ulterior grado. En este caso, coincidimos con el profesor BALLESTERO (1983) al afirmar que

debe de entenderse que el voto unipersonal no puede predicarse para las cooperativas llamadas de "segundo grado", es decir, las que tienen como socios a cooperativas en funcionamiento (de primer grado). En este caso no cabe hablar de "voto personal", pues la base asociativa es de entes societarios. Por ello en estas cooperativas de segundo grado habrá que estar al voto proporcional y no al directo que resulta lógicamente imposible (pero no olvidemos que lo practican en su "primer grado").

Lo que resulta evidente es que, a pesar de la dialéctica participación-eficiencia para el cumplimiento del principio de "un socio, un voto", éste constituye una característica netamente diferenciadora respecto a cualquier otro tipo de sociedades, cuya "democracia" se sustenta en la participación proporcional a la aportación de capital social. Sin embargo parece oportuno añadir que el cumplimiento del principio de gestión democrática no garantiza que una cooperativa sea democrática, es decir dicho cumplimiento funciona como condición necesaria no suficiente. De la misma forma, el que los ciudadanos tengamos la oportunidad de votar cada cuatro años no implica que nos encontremos en una sociedad plenamente democrática. La democracia supone una aspiración permanente de la cooperativa. Participar es algo más que cumplir unas estrictas normas de voto.

Muchos piensan que el principio hay que cumplirlo porque lo estipula la ley. Otros, que los socios no entienden de determinados asuntos: luego es inútil que participen. Otros, que la participación supone un retraso en la toma de decisiones y por tanto hace que la cooperativa sea menos competitiva. Algunos de estos argumentos tienen una justificación lógica. Pero si nos dejamos llevar más por el pragmatismo que por la convicción, al final, como venimos proponiendo insistentemente, de la cooperativa no quedará más que el nombre.

Conseguir un funcionamiento democrático es un objetivo de la cooperativa. Noes algo ya conseguido por el hecho de que se cumpla estrictamente un principio. Una cooperativa que pretenda serlo ha de establecer mecanismos de información y formación entre sus socios que permitan una participación responsable capaz de aprovechar las "iniciativas" de unos, las "prudencias" de otros y la opinión de todos. 


\subsection{Participación económica de los socios}

\begin{tabular}{|l|l|}
\hline Formulación de 1966: & $\begin{array}{l}\text { Formulación de 1995: } \\
\text { Los excedentes }\end{array}$ \\
olas economías & al capital de sus cooperativas y lo \\
eventuales resultantes & gestionan de forma democrática. \\
de las operaciones de & Al menos una parte de los activos es por \\
una sociedad pertenecen & regla general, propiedad común de la \\
a los miembros de esa & cooperativa. \\
sociedad y deberán & Normalmente reciben una compensación, \\
repartirse de forma que & si la hay, limitada sobre el capital \\
se evite que uno de & entregado como condición para ser socio. \\
entre ellos gane a & Los socios asignan fines: el desarrollo de \\
expensas de los otros. & la cooperativa; la constitución de reservas, \\
Si se paga un interés & de las cuales, al menos una parte sería \\
al capital social, su & irrepartible; el beneficio de los socios en \\
tarifa deberá ser & proporción a sus operaciones con la \\
estrictamente limitada". & cooperativa; y el apoyo de otras \\
& actividades aprobadas por los socios". \\
\hline
\end{tabular}

a) El reparto de excedentes

En la prehistoria, ante la alternativa de matar un mamut, obien quedarse sin cena, no habría tiempo de discutir cómo repartir el trabajo o asignar tareas -por cierto, conflictos frecuentes en las cooperativas-. Básicamente, quien veía el mamut desde mayor distancia era el oteador oficial, quien corría más deprisa era el corredor principal, quien arrojaba su lanza de manera más precisa era el gran tirador, y quien era más respetado y más escuchadoera el jefe. En esoconsistía todo. ¿Cuál es la razón de que todos los miembros del grupo estuvieran tan deseosos de efectuar su parte del trabajo (otear, correr, alancear, mandar) y de hacerse a un lado cada vez que alguien podía hacerlo mejor que ellos? Porque todos tenían que comer del bicho una vez muerto y asado. Lo que importaba era los resultados, no el status.

Como bien señala R.SEMLER (1991) -presidente de SEMCO, una empresa original donde las haya-, 
el beneficio de la empresa es la carne del mamut contemporáneo. Y aunque existe una visión extendida de que el reparto de beneficios constituye algún tipo de infección socialista, existen pocas herramientas motivadoras más capitalistas. Todo el mundo está de acuerdo en que los beneficios deberían ser de quien arriesga su capital, en que el comportamiento del empresario merece su recompensa, en que la creación de riqueza debiera enriquecer al creador. Bueno, dependiendo de cómo se defina capital y riesgo, todos esos tópicos pueden aplicarse tanto a los trabajadores como a los accionistas.

Desde luego el cooperativismo descubrió esto hace ya algunos años y realizó un reparto del riesgo empresarial acorde con unos valores distintos a los proclamados por el liberalismo decimonónico. Como afirma R. RAMAEKERS(1984),

la cooperación es una práctica que cuestiona, no solamente los fundamentos, sino también el ejercicio del poder económico -principio de gestión democrática- y la distribución de las ganancias -principios en torno a la ganancia- tal como existen por lo general en la inmensa mayoría de las empresas privadas o públicas.

Nos enfrentamos a una de las características netamente diferenciadoras de las sociedades cooperativas: la distribución del excedente generado, no en función del capital aportado por los socios, sino en proporción a la actividad que dichos socios realizan o, mejor dicho, aportan a la cooperativa.

Sin embargo, dadas las diversas posibilidades de reparto del beneficio (precios, excedentes e interés), su cumplimiento precisa ciertas manifestaciones que conviene matizar. BALLESTERO (1983) afirma que las cooperativas con mentalidad capitalista pueden distribuir las ganancias en proporción al capital aportado (fijo + circulante) y, en ocasiones, hasta de un modo regresivo, más que proporcional al capital. De hecho, si las cooperativas reparten el excedente cooperativo vía precios del producto entregado, el resultado es un beneficio nulo, y en consecuencia, una autofinanciación nula, una reducida o inexistente dotación a los fondos sociales...etc. Es decir, se prima el consumo, nola inversión. Lo que interesa es el corto plazo. Los cooperativistas, en el fondo, no creen en el proyecto cooperativo.

Este comportamiento típico de las cooperativas agrarias de reparto del excedente vía precios, plantea también su dificultad en las cooperativas de trabajo asociado. En este tipo de cooperativas la remuneración salarial representa 
el elemento más importante dado que la renta salarial a repartir constituye la contraprestación a la actividad cooperativizada, en este caso el trabajo.

Sin embargo podrían considerarse en esta renta dos partes: una fija, que sería una remuneración basándose en las categorías profesionales o en una valoración de puestos de trabajo; y otra variable, que sería la parte del excedente neto a repartir, variable en tanto que sería distinta para cada ejercicio, y cuyo destino lógico sería la autofinanciación o el retorno monetario. Surgen entonces dos cuestiones polémicas.

En cuanto a la parte fija: ¿qué sentido tiene en una cooperativa de trabajo asociado un abanico salarial amplio?, ¿cuál es el límite de este abanico salarial? Evidentemente estas cuestiones son cruciales en cooperativas de trabajo asociado cuya base social no es homogénea. En cualquier caso, supone una cuestión ineludible en el dinamismo de crecimiento; tanto que el aumento del abanico salarial ha sido una de las "concesiones" del Grupo Cooperativo de Mondragón a las elevadas tasas de rotación de sus altos directivos y personal especializado. El crecimiento tiene su precio... A pesar del comportamiento igualitario (9) de las cooperativas de trabajo asociado, las condiciones actuales del mercado de trabajo obligan, en cierta medida, a diferenciar económicamente a quien se diferencia en la asunción de distintas tareas y responsabilidades. La solidaridad se muestra en que tales diferencias en la remuneración fija no sean excesivas.

En cuanto a la parte variable: ¿cómo repartirla? ¿solidariamente -por partes iguales- o equitativamente -en función de la actividad, en horas por ejemplo-? Nuestra posición se sitúa en favor del criterio de la solidaridad como principio para una verdadera equidad. De esta forma la alternativa ideal sería la dotación de fondos irrepartibles. Así la cooperativa se capitaliza -aumentando la propiedad colectiva- y manifiesta su solidaridad presente y futura. Como afirma VILERT (1989),

curiosa valentía la de los cooperativistas, que anteponen la posibilidad de que otros continúen trabajando cuando ellos se jubilen, a cualquier tentación de acumulación individual. Es un reparto equitativo de beneficios entre el trabajo presente y el futuro, como una solidaridad a lo largo de la historia.

(9) Casi el setenta por ciento de las cooperativas de trabajo asociado remuneran igualitariamente a sus socios. 
b) Los intereses del capital social

Pero el criterio de reparto del beneficio tiene dos vertientes. Por una parte, la prohibición de participar como mero aportante patrimonial a una sociedad cooperativa; por otra, la limitación del beneficio al patrimonio (capital en términos socio-jurídicos) aportado por cada partícipe, que recibirá un interés fijo por tal cuota capitalista. Efectivamente, el reparto del beneficio cooperativo (excedente neto divisible) se realizará siempre en base a módulos de participación personal y nunca capitalista: si la hay, limitada sobre el capital entregado como condición para ser socio.

De acuerdo con el principio anterior, puede decirse que si la ganancia no debe desviarse hacia el capital sino hacia el trabajo, la retribución de aquél ha de ser limitada. A tal consecuencia de la identidad cooperativa se le han adherido ciertas conclusiones del todo infundadas. Una de ellas es la confusión entre "interés limitado" " "interés bajo".

Si realizamos una interpretación histórica remontándonos a los orígenes del cooperativismo, nuestra respuesta no puede ser más clara: los pioneros de Rochdale pagaban un interés del $5 \%$ cuando el tipo de interés existente en el mercado de capitales era del $2,5 \%$ (hoy día, este sistema de referencia nos situaría en tipos superiores al $16 \%$ ). Así pues, la cota que se fijaron los primeros cooperadores era el doble que la existente entonces en el mercado. Este dato refuerza la hipótesis de que la palabra "limitación" se usó desde sus orígenes como "cota" y no necesariamente como "baja cota". Esta interpretación que han utilizado la mayoría de los autores y la que han incorporado a su definición distintas legislaciones (DOMINGO SANZ, 1984, p. 25).

Por tanto, el principio cooperativo, desde este punto de vista, puede resumirse así: el capital es un medio de producción y no un fin, remunerándosele en consecuencia como tal, de acuerdo con las necesidades de la cooperativa (principio que comparten muchas PYMES). Un informe de la A.C.I. acepta como válidas las cuatro situaciones respecto a la diferencia entre el tipo de interés con el que se remunera el capital en relación con el existente en el mercado (nulo, inferior, igual o superior). La admisión de las dos últimas es obvia: responde en primer lugar, a la necesidad de no dejar a estas sociedades en inferioridad de condiciones respecto a las demás empresas, pues de lo contrario se estaría frenando el desarrollo de las empresas cooperativas, obligándolas a permanecer 
con unos moldes de actuación ampliamente superados en la actualidad por las demás sociedades (10); pero responde además a la idea original de tasa limitada lo que no significa baja.

No obstante, la doctrina de los socialistas utópicos de desviar hacia el factor trabajo las plusvalías generadas por éste, puede verse seriamente afectada, si admitimos intereses excesivamente elevados. En resumen, el capital en las sociedades cooperativas es un "medio" y no un "fin" y, por ello, se remunera de forma "fija" y no "variable"-en función de los beneficios-, lo que no implica que tal "renta" tenga que ser necesariamente "baja", aunque sí con determinados "límites" en consonancia con su carácter "no prioritario".

Pero, si no hay carne de mamut, sobran todos los principios. Los problemas de las cooperativas actuales no están en cómo repartir sino en cómo obtener(11). Los instrumentos de caza, la "forma física" -mejor la formación- de los cazadores, y aprender a cazar no sólo mamut sino también leones y conejos...son cuestiones cruciales. Pero eso es otra historia... En todo caso, la autofinanciación -y no el reparto ya sea de excedentes o de intereses- es la política coherente de cualquier cooperativa que se precie y con posibilidades de éxito (12).

(10) Resultan evidentes los problemas referentes al mayor rendimiento que pueden obtener los socios en otras inversiones alternativas, así como el problema de la recuperación de la inversión realizada en la cooperativa y la depreciación monetaria; es decir: a) El socio con recursos económicos se resistirá a autofinanciar su cooperativa en cuantía apreciable, pensando que esos mismos recursos pueden proporcionarle en el mercado de capitales beneficios más sustanciosos; b) El socio sabe que el capital que aportó al ingresar en la cooperativa o cuando ésta pidió nuevas aportaciones, se le devolverá al causar baja o al disolverse la entidad, pero con un valor real adquisitivo reducido considerablemente.

(11) Respecto a la remuneración del capital con interés limitado supone un comportamiento claramente minoritario. Sólo el once por ciento de las cooperativas de trabajo asociado españolas remuneran el capital social con un interés anual. Un análisis sectorial de este comportamiento nos aporta algunos datos significativos. El cien por cien de las cooperativas de seis sectores no remuneran el capital social: "Maquinaria", "Enseñanza", "Hostelería", "Servicios prestados a otras empresas", "Comercio al por mayor" y "Comercio al por menor". -Aproximadamente un tercio de las cooperativas de trabajo asociado de los sectores "Industria del Vidrio" y "Cuero y Calzado" remuneran el capital social. Una quinta parte de las cooperativas de trabajo asociado de los sectores "Sanidad" y "Transporte" remuneran igualmente el capital.

(12) Más de la mitad de las cooperativas de trabajo asociado españolas dedican el excedente generado -una vez dotados los fondos obligatorios- a la autofinanciación. Casi un veintiuno por ciento lo monetariza y un once por ciento lo capitaliza con el incremento de la parte correspondiente a cada socio. 


\subsection{El principio de intercooperación social y económica}

\begin{tabular}{|l|l|}
\hline Formulación de 1966: & Formulación de 1.995: \\
"Para poder servir mejor los & "Las cooperativas sirven a \\
intereses de los miembros y & a sus socios lo más \\
de la colectividad cada una & eficazmente posible y fortalecen \\
de las organizaciones deberá, & el movimiento cooperativo \\
en todas las formas posibles, & trabajando conjuntamente mediante \\
colaborar activamente con las & estructuras locales, nacionales, \\
demás cooperativas a escala & regionales e internacionales". \\
local, nacional e internacional". & \\
\hline
\end{tabular}

a) La diversidad de la intercooperación

DIVAR (1985) afirma que este principio cooperativo, impone la necesaria colaboración entre las sociedades cooperativas al efecto de que, "in solidum", mantengan la fuerza del sistema como formulación económica superadora del capitalismo, y se fortalezcan en común contra las dificultades externas. Como también señala VILERT (1989), a través de este principio el cooperativismo demuestra que, si bien nace enraizado en su realidad local, enseguida levanta sus miras para coordinarse con otras iniciativas semejantes, ampliando continuamente las nociones de espacio natural, en una visión universalista del proyecto y de la solidaridad, pero siempre sin diluir lo mas mínimo su autonomía, ni su arraigo original, que le da sentido.

Sin embargo, la realidad muestra dos ámbitos de colaboración muy diferenciados. Por un lado, su vertiente económica, que en la actualidad se designa, para todo tipo de empresas, con el término de "cooperación empresarial" (integración vertical u horizontal en cooperativas de grado superior, consorcios, grupos empresariales, etc...); por otro, su vertiente política y corporatista (PEREZ YRUELA, 1988; MOYANO ESTRADA, 1994), que se englobaría bajo el fenómeno denominado de articulación de representación de intereses a través de la acción colectiva (movimiento cooperativo o federalismo). La intercooperación económica es objeto de estudio en el ámbito de la economía de la empresa mientras que la intercooperación política entraría de lleno en el campo de la sociología. 
b) La intercooperación económica

En cuanto la cooperación de naturaleza económica entre cooperativas, RAMAEKERS (1984) señala que

sólo una solidaridad intersectorial firme y vivaz puede permitirle a la empresa cooperativa no sufrir demasiado severamente los efectos de las fuerzas económicas y sociales no cooperativas, sean éstas capitalistas o estatales. Autonomía financiera, precios competitivos, parte importante en el mercado, sólida imagen de marca, credibilidad de la cooperación como experiencia, pero también como solución: ese es el desafío que plantea la intercooperación.

La realidad nos muestra unos pocos casos aislados ejemplares (Mondragón Corporación Cooperativa, Grupo Empresarial Valenciano, ANECOOP, ASISALAVINIA,...) y multitud de casos que demuestran que el camino por recorrer es arduo, y que la intercooperación económica no es, ni mucho menos, un comportamiento típicamente cooperativo(13).

c) La intercooperación política

Nuestros comentarios van a dirigirse sobre todo a la intercooperación política. La acción colectiva ha sido objeto de un tratamiento significativo a partir del trabajo de OLSON (1965) en el que se pone especial énfasis en el tema de la cooperación entre individuos que emprenden un determinado tipo de acción para la consecución de bienes de tipo colectivo. Estos bienes están caracterizados por el hecho de ser ofrecidos globalmente y porque, una vez logrados, no es posible

(13) El once por ciento de las cooperativas de trabajo asociado españolas pertenecen a alguna cooperativa de segundo grado o alguna entidad empresarial. Por comunidades autónomas destacar: Aragón $(54,7 \%)$ y País Vasco $(27,9 \%)$ con porcentajes muy elevados de intercooperación empresarial. Superan la media nacional: $(18,5 \%)$, País Valenciano $(16,7 \%)$; Castilla La Mancha y Navarra no tienen entidades que presenten este comportamiento. Por sectores conviene resaltar los siguientes datos: Una cuarta parte de las cooperativas de trabajo asociado de los sectores "Maquinaria", "Servicios prestados a otras empresas y "Agrario" se encuentran integradas en alguna entidad de segundo grado o empresarial. Superan también la media nacional los sectores de : "Enseñanza" $(17,8 \%)$, "Industria del Vidrio" (16,2), "Corcho, Madera y Muebles" $(16,1)$ y "Limpieza" $(13,8)$. En seis sectores el grado de intercooperación es nulo: "Productos Metálicos", "Cuero y Calzado", "Sanidad", "Artes Gráficas", "Hostelería" y "Comercio al por menor". 
excluir a nadie de su disfrute (como es el caso del logro de una legislación no discriminatoria para el cooperativismo).

Nos vamos a referir, no a los aspectos teóricos de la acción colectiva, sino a la realidad concreta que se plasma en el contexto español bajo la fórmula de federaciones de cooperativas. Entre las funciones que asume el movimiento cooperativoencontramos: defender los intereses legítimos de las empresas cooperativas asociadas; representar a las cooperativas ante la Administración Pública y otras instituciones; promover, fomentary difundir la doctrina cooperativa en todos los ámbitos de la vida social, económica, cultural del territorio en cuestión; contribuir al fortalecimiento y perfeccionamiento socio-empresarial de las cooperativas asociadas mediante el asesoramiento, formación...etc; fomentar y promover las relaciones económicoempresariales entre las cooperativas comovía para alcanzarun mejor posicionamiento social y una mayor eficacia empresarial.

En el caso del movimiento cooperativo de trabajo asociado español, entre los retos planteados -con respuesta positiva al menos parcial, en algunos casospodrían citarse: desarrollo ante la Administración Pública de un papel de interlocutor permanente para aquellas cuestiones económicas y sociales que afecten directamente al sector; búsqueda de soluciones a la discriminación negativa de las cooperativas de trabajo asociado buscando la equiparación del socio-trabajador con la del trabajador por cuenta ajena de la empresa tradicional; participación en el proceso de elaboración de las leyes que le afecten; y la devolución del patrimonio histórico cooperativo.

Según el Libro Blanco de la Economía Social (1991) el $43 \%$ de las cooperativas de trabajo asociado se encuentran afiliadas a alguna de las Federaciones o Uniones (14). Si comparamos con el nivel de afiliación sindical -que podría

(14) Pero este nivel de afiliación no es homogéneo para todas las actividades productivas. Por sectores conviene resaltar dos comportamientos diferenciados. En primer lugar aquellos sectores en los que más de la mitad de las entidades integrantes se encuentran afiliadas a una federación o similar: "Sanidad" $(66,7 \%)$, "Cuero y Calzado" $(64,8 \%)$, "Enseñanza" $(61,7 \%)$, "Maquinaria" $(57,6 \%)$, "Transporte" $(57,6 \%)$ y "Comercio al por mayor" $(55,8 \%)$. En segundo lugar otro sectores donde sólo una de cada cinco cooperativas del sector de la "Construcción" y "Hostelería" se encuentran afiliadas. Además con porcentajes igualmente bajos están los sectores de "Industria de la Confección" (26,6\%), "Reparaciones" (29,4\%), "Industria del Vidrio" $(30,7 \%)$, "Servicios prestados a otras empresas" $(31,7 \%)$, "Comercio al por menor" $(36 \%)$, "Artes Gráficas" $(36,6 \%)$. 
estimarse en torno a un $15 \%$ de los trabajadores", el resultado sería netamente favorable para las cooperativas. Otra cosa sería si la afiliación se registrase no por cooperativa sino por cooperativista. En ese caso las diferencias podrían desaparecer o incluso ser desfavorables para el cooperativismo.

\section{c) Algunas reflexiones sobre el movimiento cooperativo}

Pero este indicador cuantitativo ignora algunos elementos cualitativos en los que nos interesaría insistir. Los límites de este trabajo nos obligan a optar por plantear considerandolos "sesgos" que implican nuestro conocimientode la realidad próximasóloalgunas cuestiones para la reflexión de los dirigentes del movimiento cooperativo $\mathrm{y}$ de sus afiliados, cuestiones que en algún momento merecen ser debatidas porque constituyen otros tantos retos planteados como movimiento social de acción colectiva. Entre otras podrían destacarse las siguientes:

1) Ante el peso que tiene el enfoque empresarial frente el ideológico en el actual marco del movimiento cooperativo, ¿cuáles son las ventajas e inconvenientes de la opción estructuras monoclase o sectoriales (por actividades) sobre las estructuras interclase o multisectoriales (por concepciones ideológicas)?

2) Ante la excesiva dependencia de la Administración Pública, ¿cómo ejercer acciones reivindicativas sólidas cuando se carece de autonomía?

3) Ante la heterogeneidad territorial o sectorial y amplitud de territorio por atender ¿cuáles son los mecanismos organizativos que deben articularse para ser eficaces en la prestación de servicios a los afiliados, de forma que estos perciban su utilidad?

4) Ante la existencia de recursos financieros comprometidos para acciones concretas -sobre todo en formación- y acciones prioritarias sin recursos -sobre todo en el desarrollo empresarial-, ¿ cómo ser eficaces pero también coherentes?

5) Ante la necesidad que tiene el movimiento cooperativo de alcanzar logros palpables más allá de una mera declaración de intenciones y de proyectos ¿no habría que esforzarse por trasmitir a los asociados la realidad de las acciones concretas llevadas a cabo en todos los frentes (número de cooperativas creadas, empleo generado en las cooperativas afiliadas, innovaciones, niveles de intercooperación empresarial, número y naturaleza de los servicios prestados a las cooperativas...etc)?

6) Los dirigentes del movimiento cooperativo no son los únicos responsables

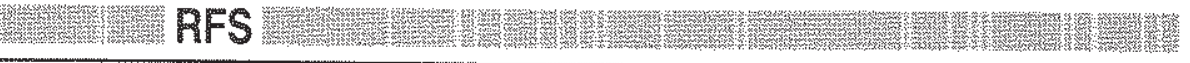


del buen o mal funcionamiento de las federaciones. Muchos fallos hay que situarlos a nivel de la propia base. De hecho la doctrina sociológica señala el problema del gorrón (free-rider), es decir, del que recibe sin hacer nada, como uno de los principales a la hora de la acción colectiva. ¿Hasta dónde son responsables las cooperativas afiliadas -y no afiliadas- de que el movimiento cooperativo no alcance los objetivos que de él se esperan?

7) ¿Es consciente el movimiento cooperativo de que el entorno -relación con las instituciones- se muestra amenazante ante actitudes protectoras de las organizaciones representativas de intereses, como son las federaciones de cooperativas?

8) Frente a la huelga para los sindicatos, frente al cierre patronal para las empresas...¿qué medios de presión tiene el movimiento cooperativo?; ¿dispone de alguno?, ¿cómo reaccionaría frente a una eventual agresión externa?

9) ¿Cuál es el precio que está dispuesto a pagar el movimiento cooperativo para mantenerse independiente del poder político?

10) Para terminar dejaremos clara nuestra postura. La intercooperación política debería caminar sobre dos "railes" o valores básicos: la autoayuda esencia del cooperativismo- y la autocrítica -esencia de la madurez y de la coherencia de una persona, pero también de las instituciones-. ¿Se encuentra el movimiento cooperativo español descarrilado?

\section{Un principio que cambia: educación, formación e información}

Formulación de 1966:

"Todas las sociedades del cooperativismo deberán constituir un fondo para impartir la enseñanza de los principios y de los métodos de cooperación, en el plano económico y democrático, a sus miembros, a sus dirigentes, a sus empleados y al gran público".
Formulación de 1995:

"Las cooperativas proporcionan educación y formación a los socios, a los representantes elegidos, a los directivos y a los empleados para que puedan contribuir de forma eficaz al desarrollo de sus cooperativas. Informan al gran público, especialmente a los jóvenes y a los líderes de opinión, de la naturaleza y beneficios de la cooperación". 
La Comisión Europea advierte que en España sólo un 15\% de la población activa recibe regularmente algún tipo de formación continua frente al $37 \%$ de la población alemana. En las grandes empresas este porcentaje aumenta hasta el $40 \%$, mientras que en las PYMES no llega al $10 \%$.

Las cooperativas no son ajenas a las crecientes necesidades de formación, sobre todo de naturaleza técnica, en un entorno cambiante (15). En numerosos trabajos, investigaciones, foros... etc se propone que la formación constituye un pilar básico para el desarrollo del cooperativismo. Los pioneros de Rochdale, al valorar este aspecto como un principio cooperativo, en cierta forma, realizaron una profecía sobre cuáles deberían ser los ejes de una empresa con futuro.

El profesor DIVAR (1983) lo interpreta en los siguientes términos: "Todo integrante de una cooperativa tiene derecho a que ésta, en la razonable medida de su correspondiente disponibilidad de fondos (a determinar mínimamente por la Ley), le asista a él y a quienes dependientemente del mismo convivan con el titular del derecho (socio o trabajador, incluido por supuesto el personal de dirección) en sus necesidades sociales, prioritariamente las educativas y de formación".

El profesor DOMINGO SANZ (1984) relativiza la importancia del principio según la formulación de 1966 por dos razones. En primer lugar, podría pensarse en la necesidad de la existencia de un fondo especial para la cobertura de determinadas necesidades atendidas insuficientemente por la Administración. De esta forma, un funcionamiento eficiente del estado acabará con la razón de ser de dicho fondo. En segundo lugar, si se dedican estos fondos de asistencia social a la formación, investigación... etc, su aplicación no sería privativa de las cooperativas, sino compartida con todo tipo de empresas.

Además, muchas cooperativas mediante determinados artilugios hacían de dudosa aplicación la letra planteada en la formulación de este principio de 1966 (16). Así por ejemplo podemos citar entre otros (BALLESTERO, 1985): cerrar a cero el ejercicio, distribuyendo beneficios vía precios e intereses, financiar las

(15) Sobre este aspecto puede verse FONDO FORMACION (1992) Las necesidades de formación en las cooperativas de Andalucía, Junta de Andalucía.

(16) Según la investigación que realizamos para la elaboración del Libro Blanco de la Economía Social casi el sesenta y cinco por ciento de las cooperativas de trabajo asociado aún no han aplicado el Fondo de Educación y Promoción Cooperativa. Aquelllas que lo han hecho le han dado en más de la mitad de los casos la formación de los socios. Otros usos han sido: ayudas a entifades culturales, deportivas,...ete, comidas de confraternización de los socios,...etc. 
actividades de una determinada minoría de los socios cooperativistas, realizar trabajos de investigación más acordes con la necesidad del incremento de productividad que la consecución de finalidades sociales... No obstante, han surgido alternativas -como la necesidad de autorización administrativa para la aplicación de dichos fondos- destinadas a la corrección de dichas desviaciones. Por tanto, a la vista de tales consideraciones, cabe dudar de la operatividad de este principio tal como esta formulado en 1966 (17).

La nueva formulación del principio pone de manifiesto la importancia del espíritu -la importancia de la formación- más que la letra -creación de un fondo- (18). Por ejemplo, si los cooperativistas no tienen formación suficiente, la participación se debilita paulatinamente, y el poderse concentra en unas pocas manos. RAMAEKERS (1984) aborda las consecuencias de este problema afirmando:

Por falta de control democrático, las cooperativas -y el sector que las agrupaevolucionarán hacia el mismo tipo de concentración de atribuciones en manos de una tecnocracia que acabará por elegir a sus dirigentes entre sus pares. Ya las asambleas de cooperativistas son silenciosas cuando no vacías, Ios consejos de administración dan su aprobación demasiado sistemáticamente... y los directores permanecen inmutables.

En nuestra opinión, el objetivo del principio cooperativo de la formación es la formación cooperativa y no cualquier tipo de formación en las cooperativas. Si se interpreta en este segundo sentido, lo que se busca es la supervivencia como empresa. Lógicamente, si no existe ningún proceso formativo, la cooperativa corre el riesgo de perder una de sus condiciones para la viabilidad como empresa a largo plazo, sea o no cooperativa.

(17) Sobre la aplicación de este principio en el caso extremeño puede verse GÓmEz CABRANES, L. (1992) "El quinto principio cooperativo". Anuario de Estudios Cooperativos, Universidad de Deusto, pp. 133-145-

(18) También en los principios de Bouchez se establecía la creación de un fondo para la formación en el segundo principio: "cada uno de ellos continuará cobrando su sueldo según la constumbre del oficio, es decir, por jornada o tarea según su habilidad individual. Se reservará una cantidad equivalente a la que los empresarios intermediarios se llevan cada jornada; a fin de año, esta cantidad que representa el beneficio neto, se dividirá en dos partes, a saber: $20 \%$ para formar o acrecer el capital social; el resto se empleará en socorro o se distribuirá entre los asociados, a prorrata de su trabajo". 
No pretendemos insistir en este asunto, aunque no vendría nada mal reflexionar hasta qué punto las cooperativas se toman en serio eso de formarse para sobrevivir o hasta dónde la formación es una necesidad sentida con mayor fuerza en los cargos del movimiento cooperativo que en el día a día de los propios cooperativistas en su empresa.

Por eso el principio habría que interpretarlo como formación cooperativa, es decir, aquella cuyos contenidos y metodología sirva para potenciar su esencia como cooperativa. En otras palabras, se precisa una reflexión sistemática y seria por parte de los que participan en el proyecto cooperativo sobre cuáles son los valores nucleares de la cooperación y cómo se está avanzando hacia ellos.

Muchos verán en esta reflexión una pérdida de tiempo. Sin embargo en la actualidad son muchas las empresas capitalistas que consideran esta reflexión vital para delimitar claramente su misión. Por esta razón conceptos como misión, visión, cultura organizativa, clima organizativo están hoy de moda (MORALES GUTIERREZ, 1995). Como en otras ocasiones, buscamos soluciones en el modelo de empresa capitalista y nos encontramos que dicho modelo sólo ofrece soluciones a problemas ya resueltos -por lo menos en sus "principios"- por el cooperativismo.

Si se acepta esta perspectiva convendría revisar seriamente qué se está impartiendo bajo el concepto de formación cooperativa. ¿Qué valores se trasmiten cuando se habla de los principios cooperativos? ¿Son estos valores coherentes con la esencia del cooperativismo? ¿No se está suponiendo, explícita o implícitamente, que el cooperativismo no tiene valores diferentes a otros modelos de empresa y que los principios no son sino reglas para cumplir con la legalidad más que para desarrollar un proyecto de empresa bajo los ejes de la soberanía de la persona y la solidaridad?

\section{Los nuevos principios}

En este apartado haremos mención de principios, que siendo implícitos en el ámbito de la cooperación, han sido explicitados aprovechando este aniversario para manifestar su importancia y su vigencia: la autonomía y el interés por la comunidad. Su relación es paradójica: las cooperativas han de ser autónomas en sus acciones y decisiones, lo que no implica que se aíslen del entorno en donde se ubican. 


\subsection{Autonomía e independencia}

\section{Formulación de 1995:}

"Las cooperativas son organizaciones autónomas de autoayuda, gestionadas por sus socios. Si firman acuerdos con otras organizaciones, incluidos los gobiernos, o si consiguen capital de fuentes externas, lo hacen en términos que aseguren el control democrático por parte de sus socios y mantengan su autonomía cooperativa".

a) La lógica de la cooperación: cooperativista y cómplice

Una de las aspiraciones de todo ser humano es la autonomía. Para los cooperativas es un síntoma de su identidad. Nos interesa reflexionar sobre determinados aspectos que, en ciertas ocasiones, hacen de la cooperativa una empresa "dependiente" interna o externamente.

Casi todos sabemos que pueden ser diversas las circunstancias que fuercen la cooperación. A veces estas circunstancias hacen ineludible unirse para superar una situación perentoria -el paro, el subdesarrollo agrario...etc-. En tales ocasiones no existe opción. Se trata de escoger entre la nada-seguir igual opeor-, y el riesgode algo que puede dar lugar a una situación más beneficiosa -mejorar-. De entre los muchos que están en circunstancias similares (19), unos pocos eligen formar una cooperativa, pero incluso entre esos pocos no todos arriesgan lo mismo.

Existen los que arriesgan (20) mucho: generalmente tienen más opciones, a corto plazo y medio plazo, dado que parten de una situación más beneficiosa cultura o bienes-. Pero también existen los gorrones: los que se aprovechan del

(19) Refiriéndonos a la procedencia de los fundadores de la empresa puede afirmarse que el sesenta y seis por ciento de las cooperativas de trabajo asociado son de nueva creación, correspondiendo casi un treinta y seis por ciento a trabajadores con anterioridad en situación de desempleo, y el resto a trabajadores que ya desempeñaban su labor por cuenta propia o ajena.

(20) Las aportaciones de los socios constituyen el principal medio de financiación de las iniciativas empresariales ctya forma social es la cooperativa de trabajo asociado, aunque esta disminuye a medida que aumenta el tamaño de la empresa pasando de un peso de casi el setenta y tres por ciento de los recursos para cooperativas de menos de ocho trabajadores a casi un cincuenta por ciento de media para cooperativas con más de cien trabajadores. 
riesgo asumido por los demás y se adhieren a la iniciativa, a remolque de los que tiran del carro. Generalmente ponen muchas objeciones, realizan muchas críticas, hacen hincapié en todos los obstáculos que hay que afrontar para que el proyecto salga adelante, pero... aportan pocas soluciones. Pero es que los que más arriesgan... ¿son unos ingenuos -y por qué no decirlo- unos tontos?

De alguna forma los que más arriesgan necesitan a los que menos: los gorrones. Los necesitan porque de alguna forma respaldan la iniciativa, son número en el proyecto,...etc. Pero la cooperación ha nacido sobre la base de unas determinadas circunstancias, no en la convicción de un proyecto empresarial coherente y solidario. El éxito-respaldado por la coyuntura externa favorable o por otros cómplices externos- puede camuflar, en muchas ocasiones, estas circunstancias y reforzar las posturas.

Son muchas las cooperativas de trabajo asociado que han nacido y se han desarrollado de esta forma. Pero esta red compleja de posturas individuales no es algo momentáneo o de los comienzos. Las situaciones críticas por las que transcurre la vida empresarial hacen aflorar la situación inicial, y tiene que darse una nueva situación de complicidad puede hacer avanzar el proyecto cooperativo...pero ¿hasta cuando?

b) Los apoyos externos y su hipoteca

La complicidad no sólo se produce en el ámbito interno. También existen cómplices externos. A la administración le interesa que el proyecto salga adelante sobre todo si ha puesto recursos o comprometido su imagen. La cooperativa se convierte entonces en un objeto de utilización política. La ayuda a fondo perdido se convierte en una hipoteca política (21). Y los cómplices internos respaldan este comportamiento: les interesa que su riesgo -inversión en recursos monetarios- sea minorado y, a ser posible, sustituido. El imprescindible riesgo empresarial se ve suplantado por el paternalismo que precisa no sólo del padre -el estado- sino también del hijo -la cooperativa-. Este papá administración puede

(21) A pesar de nuestra crítica, no conviene formarse un estereotipo del paternalismo estatal. Casi la mitad de las cooperativas de trabajo asociado españolas no han solicitado ninguna ayuda a la Administración según los datos que manejamos en el Libro Blanco de la Economía Social en España (1991). 
situarse a nivel autonómico o local. En muchos pueblos de nuestra tierra los ayuntamientos han prestado un servicio funesto en este sentido. Otras veces los agentes de desarrollo cooperativo pueden realizar la función de padres inconsciente o conscientemente; en este caso, los intereses institucionales prevalecen sobre los de las cooperativas fomentadas. Las federaciones se convierten en un fin en sí mismo en lugar de ser un medio para el desarrollo del cooperativismo. También existen padres en forma de párrocos, entidades benéficas... etc. La verdad es que sobran - ¿sobraban?-padres.

Cada cooperativa tiene unas circunstancias determinadas a las que adaptarse. Innumerables veces tendrá que sacrificar el $i d e a l$. Esto supondrá trabajar con "gorrones", negociar con padres, y sobre todo, pelear con la competencia cada vez más dura. A lo que en ningún caso debería renunciar es al proyecto cooperativo basado en la solidaridad. Ir caminando desde una cooperación de circunstancias a la cooperación por convicción.

Para la cooperativa que no tiene objetivos todos los caminos llevan a cualquier parte... es decir, que tarde o temprano de cooperativa no tendrá más que el nombre. Olvidan que no sólo tienen que vender a corto plazo, han de transformar a muchos de sus miembros de cómplices en cooperativistas.

Por el contrario, muchas cooperativas han optado por formar decididamente a sus miembros, poseen un proyecto a medio y largo plazo de lo que quieren ser no renunciando a una coherencia entre los objetivos económicos y sociales. Generalmente están sensibilizadas de que no están sólas en el ingente proyecto intercooperación-, todo ello sin descuidar, o mejor dicho, potenciando su calidad como empresas sin complejos de inferioridad respecto a otras experiencias empresariales. Hacen lo mejor posible lo que pueden hacer sin renunciar a la excelencia. Son cooperativas excelentes por que hay excelentes cooperativistas.

\subsection{Interés por la comunidad}

\section{Formulación de 1995:}

"Al mismo tiempo que se centran en las necesidades y los deseos de los socios, las cooperativas trabajan para conseguir el desarrollo sostenible de sus comunidades, según los criterios aprobados por los socios". 
Las cooperativas del valle de Mondragón fueron las primeras que hicieron una mención explícita a este principio denominándolo principio de transformación social. En efecto, una cooperativa de primer grado no puede localizarse con independencia de la actividad que llevan a cabo sus socios: ha de estar situada junto a los socios, y no en cualquier otro lugar. Ello significa una fuerte limitación, tanto más notable si la comparamos con la libertad que tiene todo empresario para elegir el emplazamiento de su empresa buscando un punto óptimo que minimice los costes o maximice la rentabilidad (BALLESTERO, 1985; p. 55).

Sin embargo, esta limitación se convierte en compromiso con su entorno, en factor de desarrollo del potencial endógeno, en un elemento de transformación social. Así, la reinversión de beneficios para la creación de puestos de trabajo, la cooperación con organismos que promuevan el desarrollo donde la cooperativa se ubica, la promoción de la cultura... etc, no son sino manifestaciones de este compromiso con la comunidad en la cual se inserta (22).

\section{Algunos principios ausentes}

\subsection{El principio mutualista}

\section{Formulación hipotética:}

"La cooperativa se compromete prioritariamente a satisfacer la necesidad de sus socios. Las operaciones con terceros no socios están supeditadas al logro de los fines sociales y a la solidaridad".

Por una parte, el principio mutualista se sostiene bajo la condición de la existencia de una necesidad colectiva previa que constituye el objeto de la cooperativa. La cooperativa se compromete a satisfacer esa necesidad de sus socios siguiendo las reglas de la doctrina cooperativa. Salirse de este ámbito-sus socios y el cooperativismo- sería una degeneración a formas empresariales frente a las cuales las cooperativas pretenden ser una alternativa. La aplicación -o no-

(22) Ver "Principios Básicos de la experiencia cooperativa de Mondragón”.Trabajoy Unión. Lankide. Noviembre, 1.987. 
del principio de mutualismo tiene repercusiones considerables. El ámbito más conflictivo -sobre todo en su vertiente fiscal- es el que hace referencia a operaciones con terceros no socios. Piénsese en la trascendencia del fenómeno en cooperativas de consumo -clientes de la cooperativa sin condición de socio-, en las cooperativas agrarias -agricultores no socios que también vende sus productos a la cooperativa-, en las cooperativas de crédito-impositores o prestatarios no socios-, o la contratación de mano de obra asalariada en las cooperativas de trabajo asociado (23).

\subsection{El principio de exclusividad}

\section{Formulación hipotética:}

Los socios de las cooperativas deben operar exclusivamente con la cooperativa en la materia objeto de contrato.

Si una vez constituida la cooperativa, los socios abandonasen en bloque esa actividad, la cooperativa perdería su objeto, y desaparecería como tal. Las cooperativas piden lógicamente a sus socios que no hagan operaciones con otras empresas competidoras, si esas operaciones van a alterar los programas de la sociedad. Se llega así a establecer un principio-de exclusividad-según el cual los socios deben operar exclusivamente con la cooperativa en la materia objeto de contrato (materia que suele llamarse actividad cooperativizada). De esta forma, si el incumplimiento de la exclusividad puede llevar a una cooperativa a la bancarrota, su cumplimiento puede colocarla en una situación de ventaja con respecto a otras empresas, al garantizar un determinado nivel de ocupación de los equipos, próximo al óptimo. Algunos ejemplos de la falta de aplicación de este principio son: cooperativistas en cooperativas agrarias que venden sus productos

(23) Bouchez (1796-1869) formuló, antes de la constitución de la experiencia clásica de Rochdale, los cuatro principios fundamentales de las cooperativas de producción a través de un artículo publicado el 17 de diciembre de 1831 en el Journal des Sciencies Morales et Politiques. Uno de estos cuatro principios esta claramente fundamentado en la hipótesis mutualista: la asociación no podrá hacer trabajar por su cuenta a obreros extraños durante más de un año; pasado este tiempo, estará obligada a admitir en su seno, el número de trabajadores nuevos que se haya hecho necesario por el crecimiento de las operaciones. 
a otras empresas; acreedores cooperativistas en cajas rurales que invierten sus recursos en otras entidades; socios trabajadores en cooperativas de trabajo asociado que desarrollan su labor en otra empresa,...

\subsection{El principio de homogeneidad}

\section{Formulación hipotética:}

La cooperativa potenciará la reducción de desigualdades entre sus socios de toda índole (social, económica,...).

Cuando hablábamos de la democracia cooperativa hacíamos una mención expresa de la necesidad de homogeneidad en la base social. El principio de homogeneidad pretende describir una situación teórica donde los socios son iguales en sus relaciones con la cooperativa. Una definición algo abstracta, pero comprensible, es la siguiente: en una cooperativa homogénea, los socios se relacionan con la sociedad a través de parámetros que toman el mismo valor, o un valor aproximadamente igual (24).

En una cooperativa poco homogénea, donde algunos socios fuertes participan junto a otros débiles y modestos, el asociacionismo puede actuar como camuflaje para que los primeros consigan cierto trato de favor al amparo de los segundos. $Y$ es que si los socios no presentan una masa homogénea a cooperativizar, resultará que su participación en consecuencia tampoco lo será. La reivindicación de poder de los socios será consecuencia de un origen donde la base social es desigual. Su devenir degenerará irremediablemente en transgresiones del funcionamiento cooperativo como pueden ser la necesidad de voto plural -en las cooperativas agrarias- o de amplias diferencias salariales -en las cooperativas de trabajo asociado-. El dinamismo cooperativo llevará, en consecuencia, a aumentar las desigualdades, en lugar de corregirlas. La homogeneización, que es tanto

(24) En otra trabajo Pérez Rojas, F., Mórales Gutuérrez, A.C. y García Puerta, J.A. (1994), realizamos un estudio empírico en cooperativas olivareras relacionando la heterogeneidad de la base social de la cooperativa con la representatividad en el consejo rector-los socios que aportan mayor cantidad de aceitunas, en este caso, serían los que ostentarian cargos de relevancia- y constatamos cierta relación. 
como decir la manifestación de la solidaridad, es una tendencia consecuente en toda cooperativa que se precie.

\section{Un principio eludido: el fenómeno cooperativo ¿es ideológicamente neutro?}

La neutralidad política del cooperativismo ha sido considerada como un principio -ayer explícito, hoy presunto-. Evidentemente conviene precisar el significado del término "neutro" pues muchos pueden estar de acuerdo en la palabra pero no en su contenido.

Si por neutralidad política se entiende el apartidismo es decir, que el cooperativismo no es patrimonio de ningún partido político, su contenido resultaría más o menos aceptable. Un partido es una organización, una forma de acción colectiva para el ejercicio del poder -o para alcanzarlo- y lógicamente el cooperativismo no puede estar supeditado a ninguna organización de este tipo (25).

En cambio, si por neutralidad política entendemos que el cooperativismo no se fundamenta en una ideología, en una forma de entender la acción, en este caso la económica, entonces nuestra posición es rotundamente negativa: así entendido, este principio ocultaría una "instrumentalización”, precisamente ideológica, del cooperativismo. El cooperativismo no puede ser políticamente "neutro" al menos por tres razones:

$\left.1^{9}\right)$ Porque opta en el ejercicio del poder interno por la participación "personalista" frente a la participación "capitalista".

La empresa capitalista basa la estructura de poder en el binomio voto-capital. Por tanto, son los promotores iniciales, o los suscriptores de capital, los que ostentan la condición de propietarios ejerciendo el poder de diversas formas: desde la dirección misma de la actividad empresarial hasta el simple control de los resultados manifestado en la mera recepción de los máximos dividendos anuales correspondientes.

La alternativa presentada por el modelo de empresa cooperativa a esta estructuración del poder en la empresa se formula sencillamente por el axioma:

(25) Experiencias recientes como la de PSV nos ofrecen un ejemplo claro de los perjuicios de la supeditación, en este caso, de una cooperativa a una organización sindical. 
"un hombre, un voto". Son las personas, por el mero hecho de serlo, quienes rigen los destinos de la sociedad independientemente del capital que aporten, que sólo les acredita como propietarios. Las aportaciones económicas dejan de ser cuotas de poder en la gestión.

$\left.2^{\circ}\right)$ Porque entiende una forma distinta de distribuir las rentas generadas por el ejercicio de la actividad: prima su contribución a la actividad en lugar de la acumulación del capital.

En la empresa capitalista, el factor determinante es la aportación de recursos económicos; por ello la ganancia se distribuye según la cuantía de los mismos. El criterio prioritario para la remuneración al capital no es su contribución a la consecución de los objetivos empresariales.

El cooperativismo, en cambio, plantea la distribución del excedente en función de la actividad realizada por los sujetos implicados en la actividad empresarial (agricultores, trabajadores, consumidores,...etc.). Se trata de primar la consecución de objetivos -la creación de riqueza, la generación de valor añadido-: en definitiva, de remunerar a los sujetos directamente implicados en la empresa, o de otra forma, de primar el factor productivo persona respecto a los restantes.

$\left.3^{\circ}\right)$ Porque opta de forma excluyente por la acción colectiva frente a la acción predominantemente individual de otras formas de actuación económica.

En efecto, en su génesis, la empresa capitalista parte generalmente de "iniciadores" individuales, los cuales mediante una idea viable y un capital suficiente emprenden la aventura de ser agentes productivos en la actividad económica. La motivación subyacente es, en la mayoría de los casos, la posibilidad de beneficiarse de la ganancia en una inversión rentable.

Sin embargo, la iniciativa empresarial no tiene por qué ser exclusivamente individual. La satisfacción de una necesidad colectiva, y de forma solidaria, puede motivar el surgimiento del emprendedor colectivo -de una acción colectiva- formado por diversos agentes económicos: agricultores, trabajadores o consumidores. De forma espontánea, intuitiva, la sociedad rompe con la inercia favorita del discurso liberalcapitalista. La iniciativa privada no depende en exclusiva de un status social predeterminado, sino que incluso no es necesario transformarse en empleador de otros para desarrollarla, democratizándose de esta forma la iniciativa empresarial (VILERT, 1989).

Por tanto, el cooperativismo se sitúa en contraposición al discurso LIBERAL (individual-capitalista) y adopta una postura ideológica SOCIAL (participativo- 
solidaria) que presenta como ejes fundamentales -valores- la primacía de la persona sobre los medios de producción y la solidaridad frente al individualismo.

¿Puede entenderse esta postura como neutral?. La respuesta contundente es que no. Pensar en una cooperativa que no participe de estos valores es pensar en lo que venimos denominando pseudocooperativismo, es decir, realidades formales-tienen el nombre de cooperativas-pero responden a un funcionamiento real mucho más cercano a la primacía del capital sobre la persona. Son un reto para el verdadero cooperativismo: supuesto que existen, hay que preguntarse cómo transformarlas; en todo caso no pueden ser consideradas dentro de lo que denominamos cooperativismo.

Desde esta perspectiva ideológica el fenómeno cooperativo está más cercano a los valores de la izquierda política que a los de la derecha. Esto resulta obvio. Una conciliación con la postura liberal sería posible: bastaría aceptar que favorecer el fenómeno cooperativo supone no oponerse a empresas que existen como unidades económicas eficientes, o que benefician de alguna u otra forma a un territorio determinado -políticas localistas-.

En cualquier caso, insistimos en que las debilidades de las cooperativas no son inherentes a la esencia de la filosofía de la cooperación sino a la forma en que se han formulado y practicado los principios cooperativos (CRAIG y SEXENA, 1984). Hemos de seguir profundizando en la esencia del cooperativismo-que tiene mucho de utopía aunque no de quimera-, sin detenernos demasiado en la letra -cuyo significado fluctúa según los intereses de quien las maneja-. 


\section{Bibliografĩa}

A.C.I. (1977); Los principios cooperativos. Centro Nacional de Educación Cooperativa. Zaragoza.

AKE Воок, S. (1990), "Cooperativas, valores fundamentales y principios cooperativos", Revista del CIRIEC-ESPAÑA, Diciembre, no 9, pp. 15-30.

BALlestero, E. (1979); "La cooperativa,"puede funcionar como una Sociedad Anónima”. Agricultura y Sociedad. $\mathrm{n}^{\mathrm{Q}}$ 10, enero-marzo, pp. 225-227.

BALlestero, E.(1983); Teoría Económica de las cooperativas. Alianza Universidad. Madrid.

BAllestero, E. (1985), Cooperativismoyempresas cooperativas.C.E.P.A.D.E., Universidad Politécnica de Madrid.

Ceña, F.; Pérez Yruela, M.; Sevilla, E. (1983); El cooperativismo agrario como forma de acción social colectiva: análisis del caso andaluz- Instituto de Sociología y Estudios Campesinos. Córdoba, Patrocinado por la Fundación Paco Natera.

Craig, J.G.; SeXENA, S.K. (1984); A critical assessment of the Co-operative Principles. Working papers, vol. $3, \mathrm{n}^{\circ} 2$. Co-operative College of Canada, Saskatoon, pp. 38-39.

Divar Garteiz-Arruecoa, J. (1985); La alternativa cooperativa. CEAC, Barcelona.

Divar Garteiz-Arruecoa, J. (1990); La democracia económica. Universidad de Deusto, Bilbao.

Domingo Sanz, J. (1984), Las empresas cooperativas en España. Algunos desarrollos teóricos Tesis Doctoral.

HesselbaCh, N. (1978); Las empresas de la economía de interés general. Siglo XXI, México.

Holyoake, J.J. (1973); Historia de los Pioneros de Rochdale. AECOOPARAGON. Zaragoza.

LAMBERT, P. (1975); La doctrina cooperativa. Intercoop. Buenos Aires, 1975.

Martínez Charterina, A. (1990); Análisis de la Integración Cooperativa, Universidad de Deusto, Bilbao.

Mateo Blanco, J. (1985); "Historia de la reforma de los principios cooperativos". REVESCO Estudios Cooperativos, nº 53, Madrid, pp. 39-58.

MonZón, J.L., BAREA, J. (Dirs.) (1991), Libro Blanco de la Economía Social en 
España, Ministerio de Trabajo y Seguridad Social, Madrid.

Monzón, J.L., Zevi, A. (Dirs.) (1994), Cooperativa, mercado, principios cooperativos, CIRIEC-ESPAÑA, Valencia.

Morales GutiérReZ, A.C. (1991), La cooperativa como realidad ideológica, social y económica, Publicaciones ETEA. Córdoba.

Morales Gutiérrez, A.C. (1993), La financiación de las cooperativas de trabajo asociado ¿problema o síntoma? Publicaciones ETEA. Córdoba.

Morales Gutiérrez, A.C. (1992), "Soberanía de la persona y solidaridad: el caso del cooperativismo", Revista de Fomento Social, $\mathrm{n}^{\circ} 185$, enero-marzo.

Morales Guttérrez, A.C. (1993), "Cooperativismo de transformación versus cooperativismo de consolidación". Anuario de Estudios Cooperativos, Universidad de Deusto, Bilbao.

Morales Gutiérrez, A.C.(1995), "La cultura organizativa: diezinterrogantes", Revista de Fomento Social, $\mathrm{n}^{\mathrm{o}}$ 198, abril-junio, pp. 193-218.

Moreau, J. (1986), "Fundamentalismo o revisionismo?". Revue des études coopératives, mutualistes et associatives". Fondation du Crédit Coopératif. Nanterre, Francia. № 17, primer trimestre.

MoyanoEstrada, E. (1994), ElasociacionismoagrarioenAndalucía.M.A.P.A..

MÜNKNER, H. (1985); Co-operative Principles and Co-operative Law. Friedrich Ebert Stiftung, Bonn.

MÜNKNER, H. (1990); "Los principios cooperativos y el progreso social", Revista del CIRIEC-ESPAÑA, Diciembre, nº 9, pp. 149-164.

NaIsBiTt, J. (1986), Reinventar la empresa. Ed. Folio, Barcelona.

Olson, J, (1965) The logic of colective action, Free press.

Pérez Rojas, F., Morales Gutiérrez, A.C. y Garcia Puerta, J.A. (1994), "El principio de homogeneidad en las cooperativas almazaras andaluzas", Geórgica, $\mathrm{n}^{\mathrm{0}}$ 2, fuera de serie.

Pérez Yruela, M. (1988), El corporativismo en España, Ed. Ariel, Barcelona. RamaEKers, R. (1984); "Análisis crítico de los principios cooperativos". Cuadernos de Economía Social. Instituto Argentino de Invest. e Información sobre Economía Coop., Solidaria y Pública. Buenos Aires, № 18, Sep-Dic. Semler, R. (1991), "Dirigir sin directivos", Harvard Deusto Business Review. THORDARSON, B. (1993), La ACl ante la reforma de los principios cooperativos", Revista del CIRIEC-ESPAÑA, Septiembre, $\mathrm{n}^{\mathrm{0}} 14$ pp. 9-15.

Vara Miranda, J. (1995), Análisis de las cooperativas de trabajo asociado de 
Madrid, M.T.S.S., Madrid.

VILERT, A. (1989), La autogestión cooperativa hoy, FCTAC, Barcelona.

WATKINS, W.P. (1986); Cooperatives principles today \& tomorrow. Holyoake Books, Manchester.

ZEV, A. (1993), "Los principios cooperativos y la financiación y las cooperativas", Revista del CIRIEC-ESPAÑA, Septiembre, $\mathrm{n}^{\circ} 14$ pp. 15-35. 